\title{
Comparación de los índices de citas y clasificación de revistas del Journal Citation Reports y Scopus en el campo de la Psicología
}

\author{
Jessica Sanmarco', María José Vázquez² y Francisca Fariña ${ }^{2 *}$ \\ ' Unidad de Psicología Forense, Facultad de Psicología, Universidad de Santiago de Compostela \\ 2 Departamento AIPSE, Universidad de Vigo
}

\begin{abstract}
- Recibido: 26 - 02 - 2019 . Aceptado: 25 - $04-2019$. Avance Online: 20 - 05 - 2019
RESUMEN: La Comisión Nacional Evaluadora de la Actividad Investigadora (CNEAl) ha tomado los índices del Journal Citation Reports y de Scopus como los criterios de referencia para la evaluación de la actividad investigadora de los profesores universitarios y del personal de las escalas científicas del CSIC, otorgando diferente valoración a cada base de datos. Estas bases proporcionan varios índices de citas para la evaluación y clasificación de las revistas y de éstos se deriva la evaluación de los investigadores. Por ello, llevamos a cabo un estudio para comparar los índices y clasificación de las revistas en ambas bases de datos. Se tomaron los índices entre los años 2012 a 2017 , Total Cites, Journal Impact Factor (JIF) y Eigenfactor Score del JCR, y SJR, CiteScore y SNIP de Scopus de todas las revistas de la categoría Psicología. Los resultados mostraron que los 3 índices de Scopus son intercambiables al compartir más del 71.4\% de la varianza y más del 64\%, con el JIF, no siendo numéricamente intercambiables. Estos resultados no están sujetos a variabilidad, permaneciendo estables. En la clasificación de revistas por cuartiles, Scopus sesga positivamente. Se discuten las implicaciones de los resultados para la evaluación de la calidad científica.
\end{abstract}

PALABRAS CLAVE: Journal Citation Reports, Scopus, Journal Impact Factor, Evaluación de la investigación, Evaluación de revistas científicas.

Comparison of the citation indexes and journal classification of the Journal Citation Reports and Scopus in the in the psychology category

ABSTRACT: The Spanish National Commission for the Assessment of the Scientific Activity (CNEAI) used the indexes from the Journal Citation Reports (JCR) and from Scopus as the reference gold standards to assess the researchers' activity, giving different value to each database. These databases provide several citation indexes for the evaluation and classification of journals from which researchers' assessment is derived. As for this, an archival study to compare the indexes and classification of journals in both databases was performed. The Total Cites, Journal Impact Factor (JIF) and Eigenfactor Score from the JCR, and SJR, CiteScore and SNIP from Scopus for a six-year period, 2012-2017, for the journals indexed in the psychology category, were recovered. The results showed that the three Scopus indexes were interchangeable sharing, at least, $71.4 \%$ of the variance, and more that $64 \%$ with the JIF, not being numerically interchangeable. As these results are not subject to variation, they will stand stable. In relation to the classification of the journals in quartiles, Scopus biasing positively. The implications of the results of the assessment of scientific quality of researchers are discussed. KEYWORDS: Journal Citation Reports, Scopus, Journal Impact Factor, Research assessment, Scientific journal assessment.

La investigación científica tiene como meta final la comunicación y difusión de los resultados

*Correspondence: Francisca Fariña.

Departamento AIPSE, Universidad de Vigo.

CP: 36005, Pontevedra, España.

E-mail: francisca@uvigo.es

(c) 2019 Sociedad Universitaria de Investigación en Psicología y Salud. Publicado por Consejo General de Colegios Oficiales de Psicólogos, España. Este es un artículo Open Access
bajo la CC BY-NC-ND licencia (http://creativecommons.org/licencias/by-nc-nd/4.0/). que generen o transfieran conocimiento, siendo el medio por antonomasia de difusión científica del conocimiento los artículos publicados en revistas científicas. Sin embargo, el número de revistas registradas como científicas es ingente (en el ISSN están registradas más de 2 millones). Dado que es evidente que no es posible una generación de tanto conocimiento 
científico para dar salida a más de dos millones de revistas, se crearon catálogos de revistas que evalúan éstas en el grado de cumplimento de criterios formales propios de revistas científicas (la Tabla 1 recoge los criterios de calidad editorial de los catálogos de la CNEAI, ANECA y LATINDEX). Ahora bien, estos catálogos también resultan insuficientes para una evaluación de la calidad científica de las revistas, pues sólo se centran en aspectos formales. En todo caso, estos catálogos, además, de los aspectos formales exigen el cumplimento de un requisito que diferencia las revistas científicas de otras revistas, la revisión por pares, generalmente en doble ciego, de los artículos allí publicados (Giri, 2019). Es tal el nivel de esta exigencia que se ha convertido en un requerimiento judicial para otorgar al conocimiento o a una técnica, la etiqueta de científica (Daubert v. Merrell Dow Pharmaceuticals, 1993). Ahora bien, estos catálogos no miden la calidad científica de los artículos y, por tanto, de las revistas. Del grado de cumplimiento de estos y valor de su contenido científico (Romero-Medina, 2009) se derivaron evaluaciones cualitativas de las revistas (p.ej., sello de calidad de la FECYT, el índice de referencia del NSD - ERIH PLUS). No obstante, el cumplimiento de estos criterios no mide, de un modo fiable y válido, la calidad de la revista. Para ello se ha recurrido a las métricas. Éstas también son muy diversas, desde las que combinan datos mixtos, cualitativos y cuantitativos (p.ej., el ICDS de MIAR que computa la inclusión en bases de datos de referencia con la pervivencia de la revista) a otras puramente cuantitativas basadas en los índices de citas. Los últimos, por su naturaleza cuantitativa, ofrecen unos resultados más fiables y válidos y con una mayor capacidad de comparación y clasificación de las revistas. Tres son las fuentes desde las que se han computado índices de citas para la evaluación de la calidad de las revistas, Web of Science $\left(W_{0}{ }^{1}\right) ; S$ ccopus $^{2}$ y Google Scholar, resultando en las bases de datos de evaluación de la calidad de revistas científicas Journal Citation Reports (JCR), Scopus Sources y Google Scholar Journal Metrics (GSJM), respectivamente.

Tabla 1

Criterios de Calidad Editorial

\begin{tabular}{|c|c|c|}
\hline CNEAI (18) & ANECA (22) & LATINDEX (33) \\
\hline $\begin{array}{l}\text { Existencia Consejo de Redacción y Comité } \\
\text { Científico }\end{array}$ & $\begin{array}{l}\text { Existencia Consejo de Redacción y Comité } \\
\text { Científico }\end{array}$ & Mención del cuerpo editorial \\
\hline Identificación miembros de los Comités & Identificación miembros de los Comités & Contenido científico \\
\hline Instrucciones detalladas a los autores & Instrucciones detalladas a los autores & Antigüedad mínima (1 año) \\
\hline Resumen & Resumen & Identificación de los autores \\
\hline Sumario (bilingüe) & Sumario (bilingüe) & Lugar de edición \\
\hline Sistema de arbitraje & Sistema de arbitraje & Entidad editora \\
\hline Datos estadísticos de la revista & Datos estadísticos de la revista & Mención del director \\
\hline Declara la periodicidad & Declara la periodicidad & Mención de la dirección \\
\hline Cumple la periodicidad & Cumple la periodicidad & Páginas de presentación \\
\hline Evaluadores externos & Evaluadores externos & Mención de periodicidad \\
\hline
\end{tabular}

\footnotetext{
' Toda la información referida a la Web of Science (WoS) está extraída de la página web https://clarivate.com/
}

${ }^{2}$ Toda la información referida a Scopus está extraída de la página web https://www.elsevier.com 
Tabla 1 (Continuación)

Criterios de Calidad Editorial

\begin{tabular}{|c|c|c|}
\hline CNEAI (18) & ANECA (22) & LATINDEX (33) \\
\hline Anonimato en la revisión externa & Anonimato en la revisión externa & Tabla de contenidos (índice) \\
\hline $\begin{array}{c}\text { Comunicación motivada de la decisión } \\
\text { editorial }\end{array}$ & Instrucciones a los evaluadores & $\begin{array}{l}\text { Membrete bibliográfico (inicio del } \\
\text { artículo) }\end{array}$ \\
\hline Apertura institucional del Comité Científico & $\begin{array}{c}\text { Comunicación motivada de la decisión } \\
\text { editorial }\end{array}$ & $\begin{array}{l}\text { Membrete bibliográfico (en cada } \\
\text { página) }\end{array}$ \\
\hline Internacionalidad del Comité Científico & Apertura institucional del Comité Científico & Miembros del Consejo Editorial \\
\hline Investigación original & Internacionalidad del Comité Científico & $\begin{array}{c}\text { Afiliación institucional de los miembros } \\
\text { del Consejo Editorial }\end{array}$ \\
\hline $\begin{array}{l}\text { Apertura institucional de los autores (Entidad } \\
\text { editora) }\end{array}$ & $\begin{array}{c}\text { Apertura institucional del Consejo de } \\
\text { Redacción }\end{array}$ & Afiliación de los autores \\
\hline Incluida en WoS/JCR y/O ERIH & Investigación original & Recepción y aceptación de artículos \\
\hline \multirow[t]{16}{*}{ Incluida en bases de datos especializadas } & Apertura institucional de los autores & ISSN \\
\hline & Incluida en WoS/JCR y/o ERIH & Definición de la revista \\
\hline & $\begin{array}{c}\text { Incluida en bases de datos especializadas } \\
+ \text { Catálogo Latindex }\end{array}$ & Sistema de arbitraje \\
\hline & Incluida en ISOC, ICYT o IME & Evaluadores externos \\
\hline & Incluida en DICE & Autores externos \\
\hline & & Apertura editorial \\
\hline & & Servicios de información \\
\hline & & Cumplimiento de la periodicidad \\
\hline & & Contenido original \\
\hline & & Instrucciones a los autores \\
\hline & & $\begin{array}{c}\text { Elaboración de las referencias } \\
\text { bibliográficas }\end{array}$ \\
\hline & & Exigencia de originalidad \\
\hline & & Resumen \\
\hline & & Resumen en dos idiomas \\
\hline & & Palabras clave \\
\hline & & Palabras clave en dos idiomas \\
\hline
\end{tabular}


La diferencia fundamental cara a la evaluación de la calidad de las revistas mediante los índices de citas radica en que mientras en la WoS y Scopus sólo se indexan documentos científicos seleccionados $y$, por tanto, los índices se computan sobre las citas recibidas de éstos, en el Google Scholar Journal Metrics se toman todo tipo de citas recibidas en cualquier documento al que tenga acceso google (esto es, no se contrasta que la cita provenga de un documento científico), e incluso con frecuencia se computa la misma cita repetidas veces. Por ello, las dos bases de datos de referencia para la evaluación de la calidad de las revistas científicas que han sido tomadas por la Comisión Nacional Evaluadora de la Actividad Investigadora (CNEAl) para la evaluación de la actividad investigadora de los profesores universitarios y del personal de las escalas científicas del CSIC, así como de las universidades para la concesión de un complemento de productividad (sexenio), son el JCR, que toma los datos de citas de la Core
Collection de la WoS, y Scopus Sources que calcula los índices a partir de los documentos indexados en Scopus (Buela-Casal, GuillénRiquelme, Ramiro-Sánchez, y Quevedo-Blasco, 2017). La WoS, que se creó en 1964, incluye referencias de publicaciones científicas desde 1900, y Scopus, disponible desde el año 2004, recoge registros desde 1996 (Moya-Anegón et al., 2007).

Para que una revista sea indexada en alguna de estas bases de datos, es imprescindible que cumpla una serie de criterios, que en gran medida son compartidos por ambas. Los criterios pueden ser mínimos o básicos y complementarios, y se agrupan en categorías, 5 en el caso de Scopus y 4 en el de WoS, aplicándose tanto factores cualitativos como cuantitativos (ver Tabla 2). El proceso de selección para la WoS/JCR es realizado por Clarivate, propietario de estas bases y sin intereses editoriales, en tanto Scopus, con intereses editoriales, lo ha externalizado al Content Selection \& Advisory Board (CSAB).

Tabla 2

Criterios de inclusión en las bases de datos Scopus y WoS/JCR

\begin{tabular}{|c|c|}
\hline Scopus & WOS/JCR \\
\hline $\begin{array}{l}\text { Política de la revista: } \\
\text { - Política editorial convincente. } \\
\text { - Tipo de revisión por pares. } \\
\text { - Diversidad geográfica de los editores. } \\
\text { - Diversidad geográfica de los autores. }\end{array}$ & $\begin{array}{l}\text { Normas básicas de publicación } \\
\text { - Revisión por pares. } \\
\text { - Prácticas de publicación éticas. } \\
\text { - Formato de Publicación: si es electrónico se evalúa si es accesible } \\
\text { para su indexación. } \\
\text { - Puntualidad: la seriedad en el cumplimiento de la periodicidad en } \\
\text { la publicación de la revista. } \\
\text { - Convenios Editoriales Internacionales } \\
\text { - Texto Completo en inglés. }\end{array}$ \\
\hline $\begin{array}{l}\text { Contenido: } \\
\text { - Contribución académica al campo de conocimiento. } \\
\text { - Claridad de los resúmenes. } \\
\text { - Calidad y conformidad con los objetivos de la revista. } \\
\text { - Legibilidad de los artículos. }\end{array}$ & $\begin{array}{l}\text { Contenido editorial } \\
\text { - Temas de publicación de la revista. } \\
\text { - Relevancia del tema en base a las citas recibidas. } \\
\text { - Tema cubierto en su base de datos. }\end{array}$ \\
\hline $\begin{array}{l}\text { Prestigio o relevancia de la revista: } \\
\text { - Citas de los artículos de la revista en Scopus. } \\
\text { - Relevancia o prestigio del editor. }\end{array}$ & $\begin{array}{l}\text { Diversidad internacional } \\
\text { - Internacionalización de los autores, los editores y el consejo } \\
\text { editorial. } \\
\text { - Se incluye un número limitado de revistas de contenidos regionales. }\end{array}$ \\
\hline $\begin{array}{l}\text { Regularidad: } \\
\text { - Publicación de la revista sin retrasos o interrupciones en el } \\
\text { calendario de publicación. } \\
\text { - Revista con ISSN registrado }\end{array}$ & $\begin{array}{l}\text { Análisis de citas } \\
\text { - Citas de los artículos de la revista en la WoS. } \\
\text { - Tasa de autocitación, debe estar como máximo en la media de las } \\
\text { revistas incluidas en la WoS. }\end{array}$ \\
\hline $\begin{array}{l}\text { Disponibilidad online: } \\
\text { - Contenido completo de la revista disponible en línea. } \\
\text { - Página principal de la revista disponible en inglés. } \\
\text { - Calidad de la página principal de la revista. }\end{array}$ & \\
\hline
\end{tabular}


A partir de ambas fuentes de datos, WoS y Scopus, se computan índices de citas de las revistas que indexan en JCR y Scopus Sources que se revisan seguidamente.

\section{- ÍNDICES DE CITAS PARA LAS REVISTAS INDEXADAS EN EL JOURNAL CITATION REPORTS}

El Journal Citation Reports (JCR) indexa más de 11,000 revistas abarcando todas las áreas de la ciencia, tecnología y ciencias sociales. De todas ellas se calculan los índices de citas Total Cites, Journal Impact Factor (JIF) y los índices derivados de éste, y el Eigenfactor Score y los índices derivados, tomando como fuente de datos la Core Collection de la Web of Science. El TC es el número total de veces que los artículos de una revista han sido citados por todas las revistas incluidas en el JCR en el año que se esté evaluando. El JIF es el número de veces que los artículos de los dos años anteriores al del cálculo del factor de impacto han sido citados en la Core Collection de la Web of Science en el año de cálculo del JCR dividido por el número de artículos publicados los dos años anteriores al del cálculo del impacto. Por ejemplo, el Factor de Impacto de 2017 de una revista $X$ sería

Citas en 2017 a los documentos publicados en 2015 y 2016 en $X$

JIF en 2017 para $X=$ Número de documentos publicados en 2015 y 2016 en X

Del JIF se derivan el Journal Impact Factor Without Self Cites (JIFwSC), el 5-Years Journal Impact Factor (5-YIF), Inmediacy Indez (II), Cited Half-Life (CH-L), y el Average Journal Impact Factor Percentile (AJIFP). El JIFwSC y el 5-YIF mantienen el mismo procedimiento de cálculo que el JIF, pero el primero no tiene en cuenta las citas que provienen de la misma publicación, y el segundo amplía los años previos de dos a cinco. Por su parte, el Inmediacy Index es la media de número de veces que un artículo es citado en el año en el que fue publicado, el Cited Half-Life es la media de edad (la edad es igual al año de publicación de los ítems citables menos el año de publicación del ítem citado) de las citas recibidas por una revista en el año de edición del JCR. El CH-L se define como la edad media de las citas producidas por una revista durante el año JCR. Finalmente, el AJIFP es el percentil que ocupa en el JIF una revista en la categoría en el año de edición del JCR.

El Eigenfactor Score se obtiene por el mismo procedimiento de cálculo que el 5-YIF, excluyendo las autocitas y dando un peso diferente a las citas acorde a la citación de la revista citante. El Normalized Eigenfactor Score es una normalización del ES, en el que la media de las revistas de la categoría es igual a 1, interpretándose como una puntuación multiplicativa de 1. Por último, el Article Influence Score mide el prestigio de la revista a partir de las citaciones recibidas durante los últimos cinco años y se obtiene dividendo el ES por el número de artículos publicados. Sucintamente, 3 son los índices de citas principales del JCR: Total Cites, Journal Impact Factor y Eigenfactor Score.

\section{-IINDICES DE CITAS PARA LAS REVISTAS INDEXADAS EN SCOPUS SOURCES}

Esta base de datos incluye más de 18.000 revistas científicas de todas las áreas del saber para las que computa índices de citas tomando como fuente los documentos indexados en Scopus. En concreto, los índices de referencia calculados son: CiteScore, el Source Normalized Impact per Paper (SNIP) y el Scimago Journal Rank (SJR), cada uno con sus índices complementarios.

El CiteScore es igual al JIF, pero para un período de tres años (se amplía a un año más la ventana de evaluación porque en el tercer año, sobre todo en Ciencias Sociales y Humanidades, la probabilidad de citación es más elevada). La fórmula de cómputo para una revista $Y$ en 2017 , sería:

$$
\text { CS en } 2017 \text { para } Y=\frac{\begin{array}{c}
\text { Citas en 2017a los documentos } \\
\text { publicados en 2014,15 y 16 en Y }
\end{array}}{\begin{array}{c}
\text { Número de documentos } \\
\text { publicados en 2014,15 y } 16 \text { en Y }
\end{array}}
$$

El CiteScore se complementa, para su correcta interpretación, con el CiteScore Percentile (posición relativa en percentiles de 
la revista en su categoría), CiteScore Rank, Quartile y Top 10\% por cada campo en el que está incluida. El CiteScore Percentile indica la posición relativa de una revista en su campo; el CiteScore Rank, el ranking en el que está la revista en su categoría); el Quartile, la clasificación de la revista en cuartiles en su categoría; y el Top 10\% Percentile, la clasificación de las revistas que están en el 10\% superior en la categoría.

EI SNIP pondera el peso de las citas por el número total de citas de la categoría, de modo que el peso es mayor en aquellas categorías con menos citas, y viceversa. Así, se normalizan las puntuaciones, permitiendo la comparación de revistas de diferentes categorías. EI SJR es similar al CiteScore, pero ponderando el peso de las citas por el valor de la revista citante y excluyendo las auto-citas. El resultado es un índice normalizado que posibilita comparar revistas de diferentes categorías. Éste índice de citas se acompaña de otros indicadores entre los que son de destacar el Cites per Document (2 years) equiparable al JIF con datos de Scopus.

En resumen, los índices de citas principales de Scopus son el CiteScore, SNIP y SJR.

\section{-EL PRESENTE ESTUDIO}

Ante esta dualidad de fuentes, WoS y Scopus, con un elenco de documentos indexados y, por tanto, de los que se obtienen las citas para los cálculos de los índices significativamente diferente $(11,000$ vs. 18,000 revistas); la diversa proliferación de índices de citas; y el valor cualitativamente diferente que se otorga a unos y otros en la evaluación de la calidad científica (mayor al JIF del JCR, que al CiteScore y SJR de Scopus), nos planteamos un estudio de archivo con el objeto de conocer el grado de concordancia en la indexación de las revistas en las bases de datos JCR y Scopus Sources; los efectos de las discrepancias en la clasificación de revistas y si los índices miden lo mismo o constructos diferentes y el grado en que están explicados por el total de citas (Salvador-Oliván y AgustínLacruz, 2015) en la categoría Psicología.

\section{MÉTODO}

\section{- OBTENCIÓN DE DATOS}

Para la realización de este estudio se han extraído los datos de los indicadores métricos de las revistas de psicología indexadas en dos bases de datos, Scopus de Elsevier y en el JCR de Clarivate Analytics. Para ello, se descargaron las listas con los indicadores de las revistas correspondientes al período de tiempo comprendido entre los años 2012-2017, un sexenio, que es la unidad de medida de la calidad de investigación de los investigadores, de ambas bases de datos durante la última semana del mes de diciembre de 2018 (no se incluye 2018 porque no está cerrado en ninguna de las bases, ni volcadas todas las revistas). Debido a las diferencias de cada base de datos en el sistema de búsqueda, el proceso de obtención de datos fue distinto para cada una de ellas:

En WoS, desde la aplicación InCites del JCR se seleccionó, en primer lugar, el año de publicación de las métricas en el campo "Select JCR Year", por lo que se realizaron 6 búsquedas para cada año del 2012 al 2017, junto con la inclusión de las bases Science Citation Index (SCI) y Social Science Citation Index (SSCI), en el campo "Select Edition". A continuación, se redujo la búsqueda a la temática de las revistas atendiendo a la clasificación de las disciplinas elaborada por Thomson Scientific (en la actualidad Clarivate Analytics) que incluyen aquellas revistas de psicología en el campo "Selected Categories": "Psychology", "Psychology Applied", "Psychology Biological", "Psychology Clinical", Psychology Developmental", "Psychology Educational", "Psychology Experimental", "Psychology Mathematical", "Psychology Multidisciplinary", "Psychology Social" y "Psychology Psychoanalysis". Por último, se descargó un fichero de datos XLS por cada año de búsqueda con los indicadores "Total Cites", "Journal Impact Factor" y "Eigenfactor Score".

En Scopus, desde la aplicación "Source", se seleccionaron todas las revistas incluidas en la Área (Subject area) "Psychology", que 
incluye las categorías "Applied Psychology", "Clinical Psychology", "Developmental and Educational Psychology", "Experimental and Cognitive Psychology", "General Psychology", "Neuropsychology and Physiological Psychology", "Psychology (miscellaneous)" y "Social Psychology", seleccionando el año en el campo"View metrics for year", de 2012 a 2017. Una vez realizada la búsqueda se descargaron los datos en un documento de XLS en el campo "Download Scopus Source List" que incluye todos los datos del período escogido, con todos los indicadores métricos, de los que se tomaron el CiteScore, el Scimago Journal Rank (SJR) y el Source Normalized Impact per Paper (SNIP) que conforman el elenco de índices de referencia para Scopus.

Para el análisis de datos se creó un fichero en el que se combinaron en una única base de datos los indicadores bibliométricos de Scopus (CiteScore, SNIP y SJR) y del JCR (Total Cites, Journal Impact Factor, Eigenfactor Score) de los años 2012 a 2017 . Posteriormente, se identificaron y eliminaron aquellas revistas que estaban duplicadas por nombre o ISSN o cuyos registros estaban vacíos, quedando un total de 1,214 revistas.

\section{-ANÁLISIS DE DATOS}

A fin de conocer el grado de consistencia inter-índices se correlacionaron todos ellos en un sexenio (unidad de medida de la calidad científica del investigador) y se computó la correlación promedio, que es un mejor estimador de la correlación verdadera que una única medida, así como los índices de dispersión (i.e., desviación típica, varianza y coeficiente de variabilidad) para estimar la estabilidad. A nivel de clasificación, correlaciones mayores de .70 se califican de altas; mayores de .80 de muy altas, y mayores de .90 como equivalentes y propias de decisiones importantes (Guilford, 1954; Nunnally, 1978). Adicionalmente y para estimar el impacto de los índices de correlación se computaron, en línea con las recomendaciones de Amado, Arce y Herraiz (2015) y Fariña, Redondo, Seijo, Novo y Arce (2017), la varianza compartida $\left(r^{2}\right)$, la independencia entre las distribuciones de las variables correlacionadas (U1), la probabilidad de superioridad de una puntuación sobre la otra correlacionada (PS), el intervalo de confianza para la media, el intervalo de credibilidad para la población y los tamaños del efecto. Para la

Tabla 3

Correlaciones entre los principales índices de citas de Scopus y el JCR

\begin{tabular}{|c|c|c|c|c|c|c|c|c|}
\hline & \multicolumn{3}{|c|}{ Scopus } & \multicolumn{3}{|c|}{ JCR } \\
\hline & & & CS & SNIP & SJR & TC & JIF & ES \\
\hline \multirow{13}{*}{ 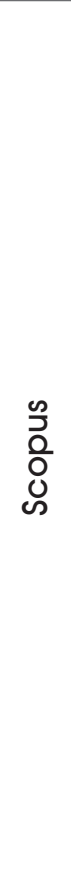 } & CS & & --- & & & & & \\
\hline & SNIP & 2012 & $.790^{\star \star \star \star}$ & ----- & & & & \\
\hline & & 2013 & $.862^{\star \star \star}$ & & & & & \\
\hline & & 2014 & $.872^{\star * \star}$ & & & & & \\
\hline & & 2015 & $.890^{* * *}$ & & & & & \\
\hline & & 2016 & $.887^{* \star *}$ & & & & & \\
\hline & & 2017 & $.906 * \star *$ & & & & & \\
\hline & SJR & 2012 & $.930^{\star \star *}$ & $.797 * * *$ & ----- & & & \\
\hline & & 2013 & $.930^{* * *}$ & $.842^{\star \star \star}$ & & & & \\
\hline & & 2014 & $.928^{* * *}$ & $.841^{* \star *}$ & & & & \\
\hline & & 2015 & $.939 * \star *$ & $.852^{\star \star \star}$ & & & & \\
\hline & & 2016 & $.946 * \star \star *$ & $.853^{\star \star \star}$ & & & & \\
\hline & & 2017 & $.949 * \star *$ & $.885^{\star \star *}$ & & & & \\
\hline
\end{tabular}


Tabla 3 (Continuación)

Correlaciones entre los principales índices de citas de Scopus y el JCR

\begin{tabular}{|c|c|c|c|c|c|c|c|c|}
\hline & & & & Scopus & & & JCR & \\
\hline & & & CS & SNIP & SJR & $\mathrm{TC}$ & JIF & ES \\
\hline & TC & 2012 & $.643^{* * *}$ & 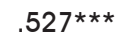 & $.665^{\star * \star}$ & ----- & & \\
\hline & & 2013 & $.598 * \star \star$ & .491 *** & $.649 * * *$ & & & \\
\hline & & 2014 & $.583^{* * *}$ & $.491 * \star *$ & $.635^{\star \star *}$ & & & \\
\hline & & 2015 & $.551 * * *$ & $.457 * * \star$ & $.623^{* * *}$ & & & \\
\hline & & 2016 & $.569 * \star \star$ & $.462 * \star \star$ & $.635^{\star * *}$ & & & \\
\hline & & 2017 & $.555^{\star \star \star}$ & $.455^{\star * *}$ & $.603^{* * *}$ & & & \\
\hline & JIF & 2012 & 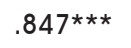 & $.854 * \star \star$ & $.802 * \star \star$ & $.593^{* * *}$ & ------- & \\
\hline & & 2013 & $.859 * * \star$ & $.797^{\star * \star}$ & $.832^{* * *}$ & $.555^{\star \star \star}$ & & \\
\hline & & 2014 & $.820 * \star \star$ & $.829 * * \star$ & $.769 * * \star$ & $.540 * * *$ & & \\
\hline$\underline{\sim}$ & & 2015 & $.829 * * \star$ & $.792 * * *$ & $.785^{\star \star *}$ & $.523 * \star *$ & & \\
\hline & & 2016 & $.839 * * *$ & $.786 * * *$ & $.820 * * *$ & $.535^{\star * \star}$ & & \\
\hline & & 2017 & $.779 * * \star$ & $.799 * * *$ & $.814^{\star \star *}$ & $.436 * * *$ & & \\
\hline & ES & 2012 & $.694^{* * *}$ & $.501 * * \star$ & $.703^{* * *}$ & $.879 * * *$ & $.629 * * *$ & -----. \\
\hline & & 2013 & $.643^{* * *}$ & $.468 * * *$ & $.468^{* * *}$ & $.882 * * *$ & $.605^{* \star *}$ & \\
\hline & & 2014 & $.647 * \star *$ & $.480 \star * \star$ & $.670 * * *$ & $.870 * \star \star$ & $.588 * \star *$ & \\
\hline & & 2015 & $.591 * \star \star$ & .426 *** & $.640 * * *$ & $.834^{\star \star \star}$ & $.554 * \star \star$ & \\
\hline & & 2016 & $.553^{\star \star \star}$ & $.383^{* * *}$ & $.599 * * *$ & $.800 * * *$ & $.518^{* * *}$ & \\
\hline & & 2017 & $.500 * \star \star$ & $.359 * * *$ & 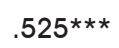 & $.779 * * *$ & $.422 * \star \star$ & \\
\hline
\end{tabular}

transformación de los tamaños del efecto se tomaron la Tablas de Salgado (2018) y para su interpretación se siguió la transformación en percentiles y porcentaje de superioridad (Monteiro, Vázquez, Seijo, y Arce, 2018; Vilariño, Amado, Vázquez, y Arce, 2018).

\section{RESULTADOS}

\section{- CORRELACIONES DE LOS ÍNDICES PRINCIPALES DE CITAS DE SCOPUS Y DEL JCR}

La correlación entre los tres índices principales de Scopus es muy elevada (ver Tabla 3 por años: límite inferior de .790 , es decir, comparten como mínimo el $62.41 \%$ de la varianza; límite superior de .949, o sea, comparten el $90.1 \%$ de la varianza). Más explícito aún es el promedio de las correlaciones entre dichos índices de citas (ver Tabla 4), siendo del $71.4 \%$ la varianza compartida entre SNIP y SJR; del $75.3 \%$ entre CiteScore y SNIP; y del $87.8 \%$ entre CiteScore y SJR. En suma, y dado que provienen de la misma fuente (base de datos) se podría sostener que estos indicadores son intercambiables y que el CiteScore y el SJR son el mismo, añadiendo este último que permite la comparación entre revistas de diferentes categorías al ser una puntuación normalizada. Además, el control de las autocitas que diferencia al SJR resulta irrelevante al ser intercambiable este índice con el CiteScore que no descarta en el cómputo las autocitas. A nivel práctico de evaluación 
de la calidad de las revistas se debería, por tanto, tomar el más beneficioso en caso de discrepancia en la evaluación cualitativa de las mismas.

Por su parte, los índices de citas derivados de los datos del JCR no son todos homogéneos. Así, el Journal Impact Factor no es el Total de citas $(r<.70,28.1 \%$ de varianza compartida); ni el Journal Impact Factor, el Eigenfactor Score (30.6\% de varianza compartida). No obstante, el Eigenfactor Score está muy altamente explicado $(r>$.80) por el Total de Citas $(70.7 \%$ de varianza compartida).

Finalmente, los contrastes inter-índices de Scopus y del JCR advierten que los tres índices de citas de Scopus, que recordemos son muy semejantes entre sí, comparten medida con el Journal Impact Factor (varianza compartida con el Journal Impact Factor: $68.7 \%$ el CiteScore; $65.4 \%$ el SNIP; y $64.6 \%$ el SJR). No obstante, estos índices no solapan su distribución con el JIF, U1 $=.925, .907$ y .903 con el CiteScore, SNIP y SJR, respectivamente. En otras palabras, las distribuciones son independientes en el 92.5\% (CiteScore), $90.7 \%$ (SNIP) y $90.3 \%$ (SJR), del JIF. Esto se debe a que la puntuación en el CiteScore es superior en el 98.1\%, PS $=.981$, de los casos a la del JIF, en tanto la del SNIP y del SJR es inferior, PS $=.974$ y .972 , en el $97.4 \%$ y el $97.2 \%$. Sucintamente, las evaluaciones de los índices de citas de Scopus están altamente asociadas con el JIF, pero son numéricamente diferentes. Además, destaca que ninguno de los índices de Scopus está explicado significativamente por el Total de Citas (el límite superior del intervalo de confianza para la correlación media es inferior a.70).

Conocida esta relación inter-índices de citas, se hacía necesario saber si estos resultados son invariables o están sujetos a variabilidad. El coeficiente de variabilidad, así como los valores de la varianza muy cercanos a cero (ver Tabla 4), ponen de manifiesto que los resultados son altamente estables en todos los índices correlacionados. Aún es más, el intervalo de confianza para el valor de credibilidad muestra que, de no mediar cambio significativo alguno, estos datos serán estables en el tiempo (el límite inferior es el valor mínimo que podría darse y el superior el máximo con una probabilidad asociada del 95\%). A nivel de evaluación, los datos permiten sostener que el CiteScore y el SJR miden por igual ( $r$ $>$.90; $87.8 \%$ de varianza compartida); que el grado de consistencia en la evaluación entre el CiteScore y el SJR es significativamente superior (los intervalos de confianza para la media no se solapan y es mayor para CiteScore con el SJR) al hallado entre CiteScore y SNIP y entre SNIP y SJR, en tanto que la consistencia entre CiteScore y SNIP, y SNIP y SJR es muy alta $(r$ $>$.80). No obstante, las distribuciones del CiteScore y del SJR son independientes, U1 $=.996$ (probabilidad de no solapamiento entre las dos distribuciones), siendo el impacto numérico superior (valores más altos), PS = .9999, en el CiteScore que en el SJR. Así pues, los valores numéricos del CiteScore y el SJR no son intercambiables. En consecuencia, si la diferencia entre CiteScore y el SJR es que el SJR tiene en cuenta en el cómputo el valor de la fuente (no todas las citas pesan igual, en tanto en el CiteScore todas tienen el mismo peso), los resultados ponen de manifiesto que, si bien numéricamente ambos índices son distintos, existe una correspondencia casi total entre ellos de modo que el efecto del valor de la fuente en el índice es prácticamente nulo.

\section{- CLASIFICACIÓN DE REVISTAS}

La Tabla 5 resume el número de revistas por año incluidas en cada base de datos y el grado de solapamiento. Los datos advierten que Scopus $(n=1,081)$ indexa un número significativamente mayor, $\chi^{2}(1)=122.49, p<$ .001 , de revistas que el JCR $(n=624)$. A su vez, el grado de solapamiento de las revistas indexadas en ambas bases de datos no llega al $50 \%$ del total, $M=46.9 \%$, estando en torno al $15 \%, M=14.4 \%$, de las indexadas en el JCR, no indexadas en Scopus; y ligeramente más del $50 \%, M=51.1 \%$, de las indexadas en Scopus, no están en el JCR. El número total de revistas indexadas se eleva a 1,171 con un crecimiento anual promedio del $2.55 \%$.

Las revistas propias del JCR se distribuyen uniformemente por los cuartiles, $\chi^{2}(3)=6.00$, ns, pero no así las de Scopus, $\chi^{2}(3)=127.96$, 
Tabla 4

Media y variabiliad en la correlación entre índices de citas

\begin{tabular}{|c|c|c|c|c|c|}
\hline & $M$ & $\mathrm{IC}_{\mathrm{M}} 95 \%$ & $V_{P} 95 \%$ & $S^{2}$ & $\mathrm{CV}$ \\
\hline \multicolumn{6}{|c|}{ Índices de citas de Scopus } \\
\hline CS con SNIP & .868 & {$[.834, .901]$} & {$[.787, .948]$} & 0.002 & 4.730 \\
\hline CS con SJR & .937 & {$[.930, .944]$} & {$[.919, .955]$} & 0.000 & 0.964 \\
\hline SNIP con SJR & .845 & {$[.822, .868]$} & {$[.789, .901]$} & 0.001 & 3.365 \\
\hline \multicolumn{6}{|c|}{ Índices de citas del JCR } \\
\hline TC con JIF & .530 & {$[.496, .564]$} & {$[.428, .632]$} & 0.003 & 9.835 \\
\hline TC con ES & .841 & {$[.806, .876]$} & {$[.755, .926]$} & 0.002 & 5.196 \\
\hline JIF con ES & .553 & {$[.498, .607]$} & {$[.406, .699]$} & 0.006 & 13.562 \\
\hline \multicolumn{6}{|c|}{ Inter-índices de citas del JCR y Scopus } \\
\hline CS con TC & .583 & {$[.556, .610]$} & {$[.516, .650]$} & 0.001 & 5.855 \\
\hline CS con JIF & .829 & {$[.806, .851]$} & {$[.774, .884]$} & 0.001 & 3.372 \\
\hline CS con ES & .605 & {$[.548, .661]$} & {$[.466, .743]$} & 0.005 & 11.707 \\
\hline SNIP con TC & .480 & {$[.458, .503]$} & {$[.425, .535]$} & 0.001 & 5.833 \\
\hline SNIP con JIF & .809 & {$[.788, .831]$} & {$[.758, .861]$} & 0.001 & 3.261 \\
\hline SNIP con ES & .436 & {$[.391, .481]$} & {$[.325, .547]$} & 0.003 & 12.979 \\
\hline SJR con TC & .635 & {$[.618, .652]$} & {$[.593, .677]$} & 0.000 & 3.351 \\
\hline SJR con JIF & .804 & {$[.785, .822]$} & {$[.758, .849]$} & 0.001 & 2.907 \\
\hline SJR con ES & .601 & {$[.548, .653]$} & {$[.425, .776]$} & 0.008 & 14.915 \\
\hline
\end{tabular}

Nota. M: Media; IC $95 \%$ : Intervalo de confianza para la media al 95\%; VC $95 \%$ : Intervalo del valor de credibilidad al 95\%; S2: varianza; CV: Coeficiente de variabilidad.

Tabla 5

Número de revistas incluidas en Scopus y JCR por año

\begin{tabular}{|c|c|c|c|c|}
\hline Año & Recistas Scopus & Revistas JCR & $\begin{array}{l}\text { No total revistas } \\
\text { (Scopus \& JCR) }\end{array}$ & $\begin{array}{c}\% \text { incremento año } \\
\text { anterior }\end{array}$ \\
\hline 2017 & $\begin{array}{c}1081 \\
\text { 547(50.6\%): Scopus } \\
534(49.4 \%): \text { JCR }\end{array}$ & $\begin{array}{c}624 \\
534(85.6 \%): \text { Scopus } \\
90(14.4 \%): \text { JCR }\end{array}$ & $\begin{array}{c}N=1171 \\
n_{\text {scopus }} \& J C R=534 \\
\% \text { scopus } \& J C R 45.6 \%\end{array}$ & $\begin{array}{l}\text { Total: } 4.01 \% \\
\text { Scopus: } 4.1 \% \\
\text { JCR: } 2.9 \%\end{array}$ \\
\hline 2016 & $\begin{array}{c}1037 \\
518(49.9 \%): \text { Scopus } \\
519(50.1 \%): \text { JCR }\end{array}$ & $\begin{array}{c}606 \\
519(85.6 \%): \text { Scopus } \\
87(14.4 \%): \text { JCR }\end{array}$ & $\begin{array}{c}N=1124 \\
n_{\text {scopus }} \& J C R=519 \\
\% \text { scopus } \& J C R 46.2 \%\end{array}$ & $\begin{array}{l}\text { Total: } 1.33 \% \\
\text { Scopus: } 1.7 \% \\
\text { JCR: }-0.002\end{array}$ \\
\hline 2015 & $\begin{array}{c}1019 \\
502(49.3 \%): \text { Scopus } \\
517((50.7 \%): \text { JCR }\end{array}$ & $\begin{array}{c}607 \\
517(85.2 \%): \text { Scopus } \\
90(14.8 \%): J C R\end{array}$ & $\begin{array}{c}N=1109 \\
n_{\text {scopus }} \& \text { JCR }=517 \\
\% \text { scopus } \& J C R 46.6 \%\end{array}$ & $\begin{array}{l}\text { Total: } 2.71 \% \\
\text { Scopus: } 2.3 \% \\
\text { JCR: } 1.8 \%\end{array}$ \\
\hline 2014 & $\begin{array}{c}996 \\
\text { 483(48.5\%): Scopus } \\
513(51.5 \%): \text { JCR }\end{array}$ & $\begin{array}{c}596 \\
86.1 \% \text { en Scopus } \\
83(13.9 \%): \text { JCR }\end{array}$ & $\begin{array}{c}N=1079 \\
n_{\text {scopus }} \& J C R=513 \\
\% \text { scopus } \& J C R 47.5 \%\end{array}$ & $\begin{array}{l}\text { Total: } 2.13 \% \\
\text { Scopus: } 2.4 \% \\
\text { JCR: } 2.0 \%\end{array}$ \\
\hline 2013 & $\begin{array}{c}972 \\
\text { 472(48.6\%): Scopus } \\
500(51.4 \%): \text { JCR }\end{array}$ & $\begin{array}{c}584 \\
85.6 \% \text { en Scopus } \\
84(14.4 \%): \text { JCR }\end{array}$ & $\begin{array}{c}N=1056 \\
n_{\text {scopus }} \& J C R=500 \\
\% \text { scopus } \& J C R \quad 47.3 \%\end{array}$ & $\begin{array}{l}\text { Total: } 2.56 \% \\
\text { Scopus: } 2.4 \% \\
\text { JCR: } 0.007 \%\end{array}$ \\
\hline 2012 & $\begin{array}{c}946 \\
449(47.5 \%): \text { Scopus } \\
497(52.5 \%): \text { JCR }\end{array}$ & $\begin{array}{c}580 \\
85.7 \% \text { en Scopus } \\
\text { 83(14.3\%): JCR }\end{array}$ & $\begin{array}{c}N=1029 \\
n_{\text {scopus }} \& J C R=497 \\
\% \text { scopus } \& \text { JCR } 48.3 \%\end{array}$ & ----- \\
\hline
\end{tabular}


Tabla 6

Distribución de revistas propias de cada base de datos por cuartil

\begin{tabular}{|c|c|c|c|c|c|c|}
\hline & & Q1 & Q2 & Q3 & Q4 & Total \\
\hline Scopus & 2017 & $57(10.42 \%)$ & $92(16.82 \%)$ & $169(30.90 \%)$ & $229(41.86 \%)$ & 547 \\
\hline JCR & & $20(22.22 \%)$ & $23(25.56 \%)$ & $22(24.44 \%)$ & $25(27.78 \%)$ & 90 \\
\hline Scopus & 2016 & $46(8.88 \%)$ & $93(17.95 \%)$ & $161(31.08 \%)$ & $218(42.08 \%)$ & 518 \\
\hline JCR & & $19(21.84 \%)$ & $16(18.39 \%)$ & $21(24.14 \%)$ & $31(35.63 \%)$ & 87 \\
\hline Scopus & 2015 & $44(8.76 \%)$ & $89(17.73 \%)$ & $158(31.47 \%)$ & $211(42.03 \%)$ & 502 \\
\hline JCR & & $14(15.56 \%)$ & $18(20.00 \%)$ & $23(25.56 \%)$ & $35(38.89 \%)$ & 90 \\
\hline Scopus & 2014 & $42(8.70 \%)$ & $88(18.22 \%)$ & $146(30.23 \%)$ & $207(42.86 \%)$ & 483 \\
\hline JCR & & $15(18.07 \%)$ & $13(15.66 \%)$ & $29(34.94 \%)$ & $26(31.33 \%)$ & 83 \\
\hline Scopus & 2013 & $39(8.26 \%)$ & $92(19.49 \%)$ & $136(28.81 \%)$ & $205(43.43 \%)$ & 472 \\
\hline JCR & & $14(16.67 \%)$ & $12(14.29 \%)$ & $30(35.71 \%)$ & $28(33.33 \%)$ & 84 \\
\hline Scopus & 2012 & $43(9.58 \%)$ & $81(18.04 \%)$ & $126(28.06 \%)$ & $199(44.32 \%)$ & 449 \\
\hline JCR & & $13(15.66 \%)$ & $15(18.07 \%)$ & $25(30.12 \%)$ & $30(36.14 \%)$ & 83 \\
\hline Scopus & Promedio & $45(9.12 \%)$ & $89(18.01 \%)$ & $149(30.16 \%)$ & $212(42.71 \%)$ & 495 \\
\hline JCR & & $16(18.38 \%)$ & $16(18.76 \%)$ & $25(29.01 \%)$ & $29(33.85 \%)$ & 86 \\
\hline
\end{tabular}

$p<.001$ (ver contingencias y porcentajes de distribución promedio en Tabla 6). Los contrastes de la distribución observada y la esperada por cuartil en las revistas propias de Scopus en la clasificación de esta misma base de datos advierten de una infra-clasificación en el cuartil $1, \chi^{2}(1)=36.93, p<.001$, y una sobre-clasificación en el cuartil $4, \chi^{2}(1)$ $=23.405, p<.001$. En concreto, las revistas propias de Scopus tienen una probabilidad 1.70 veces, $\mathrm{OR}=1.70$, mayor de estar clasificadas en el cuartil 4 (percentil 60.64\%, $\mathrm{P}_{60.64}$, es decir, mayor que el $21.28 \%$ de todos los tamaños del efecto positivos posibles) que la esperada, y 2.74 veces menos, $O R=0.36$ (percentil 65.17\%, $\mathrm{P}_{65.17}$, es decir, mayor que el $30.34 \%$ de todos los tamaños del efecto positivos posibles) en el cuartil 1 .

Esta infra- y sobre -clasificación en los cuartiles 1 y 4 de las revistas propias de Scopus se refleja en la clasificación cuartílica en las revistas comunes a ambas bases de datos (ver Tabla 7). Así, mientras la tasa de clasificación de revistas es uniforme inter-cuartiles en el JCR, $\chi^{2}(3)=1.00$, ns, no lo es en Scopus, $\chi^{2}(3)=$ $36.93, p<.001$. En concreto, Scopus clasifica en el cuartil 1 más revistas de las esperadas por azar, $\chi^{2}(1)=23.41, p<.001$, y menos en el cuartil 4, $\chi^{2}(1)=50.19, p<.001$. De hecho, Scopus clasifica en el cuartil 11.70 veces, $\mathrm{OR}=1.70$, más revistas de las esperadas (percentil 60.64\%, $P_{60.64}$, es decir, mayor que el $21.28 \%$ de todos los tamaños del efecto positivos posibles), y 3.57 veces menos en el cuartil 4 (percentil 69.15\%, $P_{69.15}$, es decir, mayor que el $38.3 \%$ de todos los tamaños del efecto positivos posibles), $O R=0.28$. Así pues, la clasificación de revistas de Scopus está sesgada positivamente en comparación con la procedente del JCR. Esta tendencia se ha ido acentuando con el paso de los años, tal y como lo atestigua el incremento significativamente mayor, $Z=4.001, p<.001$, de nuevas incorporaciones en Scopus, $12.5 \%$, frente a las registradas en el JCR, $7.1 \%$.

\section{DISCUSIÓN}

De los resultados descritos se derivan las siguientes conclusiones:

Los índices de citas CiteScore, SNIP y SJR de Scopus son altamente coincidentes en la evaluación de las revistas, siendo el SJR y el CiteScore prácticamente iguales. En consecuencia, la corrección por la importancia 
Tabla 7

Distribución de revistas compartidas por ambas bases de datos por cuartil

\begin{tabular}{|c|c|c|c|c|c|c|}
\hline Base & Año & Q1 & Q2 & Q3 & Q4 & Total \\
\hline Scopus & 2017 & 227 (42.51\%) & $164(30.71 \%)$ & $108(20.22 \%)$ & 35 (6.55\%) & 534 \\
\hline $\mathrm{JCR}$ & & $132(24.72 \%)$ & $142(26.59 \%)$ & $128(23.97 \%)$ & $132(24.72 \%)$ & \\
\hline Scopus & 2016 & $223(42.97 \%)$ & $167(32.18 \%)$ & 92 (17.73\%) & 37 (7.13\%) & 519 \\
\hline $\mathrm{JCR}$ & & $128(24.66 \%)$ & 140 (26.97\%) & $126(24.28 \%)$ & 125 (24.08\%) & \\
\hline Scopus & 2015 & 217 (41.97\%) & 164 (31.72\%) & 99 (19.15\%) & 37 (7.16\%) & 517 \\
\hline $\mathrm{JCR}$ & & 132 (25.53\%) & $138(26.69 \%)$ & 124 (23.98\%) & $123(23.79 \%)$ & \\
\hline Scopus & 2014 & $217(42.30 \%)$ & $158(30.80 \%)$ & 100 (19.49\%) & $38(7.41 \%)$ & 513 \\
\hline $\mathrm{JCR}$ & & 131 (25.54\%) & 137 (26.71\%) & $122(23.78 \%)$ & $123(23.98 \%)$ & \\
\hline Scopus & 2013 & 217 (43.40\%) & $140(28.00 \%)$ & 111 (22.20\%) & $32(6.40 \%)$ & 500 \\
\hline $\mathrm{JCR}$ & & $127(25.40 \%)$ & $134(26.80 \%)$ & $116(23.20 \%)$ & $123(24.60 \%)$ & \\
\hline Scopus & 2012 & 205 (41.25\%) & $143(28.77 \%)$ & $108(21.73 \%)$ & 41 (8.25\%) & 497 \\
\hline $\mathrm{JCR}$ & & $128(25.75 \%)$ & 132 (26.56\%) & 116 (23.34\%) & 121 (24.35\%) & \\
\hline Scopus & Promedio & $218(42.40 \%)$ & $156(30.39 \%)$ & $103(20.06 \%)$ & 37 (7.14\%) & 513 \\
\hline $\mathrm{JCR}$ & & $130(25.26 \%)$ & $137(26.72 \%)$ & $122(23.77 \%)$ & 125 (24.25\%) & \\
\hline
\end{tabular}

de la fuente de la cita que realiza el SJR no tiene efecto alguno en el cálculo del índice, al igual que la normalización (SNIP) por el potencial de citas en el campo temático. A nivel de evaluación cuantitativa del impacto de las revistas, estos índices se podrían tomar indistintamente, y, como es tendencia en evaluación de la calidad, asumir el más beneficioso para la evaluación de la revista. Sin embargo, el SJR y el SNIP permiten la comparación de revistas de diferentes categorías, al ser índices con puntuaciones normalizadas.

De los índices del JCR, el JIF es independiente del Total de Citas, es decir, el JIF corrige, de facto, el total de citas recibidas por el número de artículos publicados. Sin embargo, el Eigenfactor Score está explicado, en gran medida (>70\%), por el Total de Citas. En consecuencia, la corrección por el valor de la fuente y el control de las autocitas del Eigenfactor Score no se refleja en una mayor validez del índice computado. Estos resultados prestan apoyo a una mayor validez del JIF frente al ES, que está más explicado por el Total de citas.

Los tres índices de citas de Scopus presentan un alto grado de solapamiento con el JIF, tal que podrían sustituir al JIF.
Scopus indexa más revistas que el JCR, de modo que sólo aproximadamente la mitad de las indexadas por Scopus lo están también en el JCR.

Las revistas propias de Scopus están sobre-representadas en el cuartil 4 e infrarepresentadas en el cuartil 1. De este modo, las revistas comunes están sesgadas positivamente en la clasificación por cuartiles, por lo que, en caso de discrepancia, prevalece la clasificación del JCR.

Del mismo modo, la clasificación de las revistas indexadas en Scopus y no en el JCR no es válida, pero se podrían clasificar en el JCR transformando los índices de Scopus al JIF (p.ej., en puntuaciones normalizadas, tal que pueda conocerse la clasificación correspondiente en el JIF derivada de los índices de Scopus), ya que son medidas equivalentes. Esto resolvería el problema de la falta de consistencia en la indexación puesto que no se conocen razones de calidad para dicha inconsistencia.

Por su parte, en torno al 15\% de las revistas indexadas en el JCR no lo están en Scopus. Éstas se distribuyen homogéneamente por los cuartiles de modo que no sesgan la evaluación en cuartiles de las revistas comunes, resultando también su clasificación válida. 
En resumen, el JIF es el índice de medida de la evaluación de las revistas más válido; y la indexación y clasificación de revistas del JCR son más válidas que las de Scopus.

Estos resultados y, por extensión, las conclusiones, son de revistas del campo de la Psicología con lo que la generalización, aunque probablemente correcta (soporte dado por los intervalos de credibilidad), requiere de verificación.

\section{- Conflicto de intereses}

Los autores declaran no tener ningún conflicto de intereses.

\section{REFERENCES}

Amado, B. G., Arce, R., y Herraiz, A. (2015). Psychological injury in victims of child sexual abuse: A meta-analytic review. Psychosocial Intervention, 24(1), 49-62. https://doi. org/10.1016/i.psi.2015.03.002

Buela-Casal, G., Guillén-Riquelme, A., RamiroSánchez, T., y Quevedo-Blasco, R. (2017). Ranking de investigación de las universidades públicas españolas [Research ranking of Spanish public universities]. Revista Iberoamericana de Psicología y Salud, 8(1), 21-35. https://doi. org/10.23923/i.rips.2017.08.003

Daubert v. Merrell Dow Pharmaceuticals, Inc., 113 S. Ct. 2786 (1993).

Fariña, F., Redondo, L., Seijo, D., Novo, M., y Arce, R. (2017). A meta-analytic review of the MMPI validity scales and indexes to detect defensiveness in custody evaluations. International Journal of Clinical and Health Psychology, 17, 128-138. http://dx.doi. org/10.1016/i.ijchp.2017.02.002

Giri, R. (2019). Influence of selected factors in journals' citations. Aslib Journal of Information Management, 71(1), 90-104. https://doi. org/10.1108/AJIM-07-2017-0170

Guilford, J. P. (1954). Psychometric methods. New York, NY: McGraw-Hill.

Monteiro, A., Vázquez, M. J., Seijo, D., y Arce, R. (2018). ¿̇Son los criterios de realidad válidos para clasificar y discernir entre memorias de hechos auto-experimentados y de eventos vistos en vídeo? [Are the reality criteria valid to classify and to discriminate between memories of self- experienced events and memories of videoobserved events?] Revista Iberoamericana de Psicología y Salud, 9(2), 149-160. https://doi. org/10.23923/i.rips.2018.02.020

Moya-Anegón, F., Chinchilla-Rodríguez, Z., Vargas-Quesada, B., Correa- Álvarez, E., Muñoz-Fernández, F. J., González-Molina, A., y Herrero - Solana, A. (2007). Coverage analysis of Scopus: A journal metric approach. Scientometrics, 73(1), 53-78. https://doi. org/10.1007/s $11192-007-1681-4$

Nunnally, J. C., (1978). Psychometric theory. New York, NY: McGraw-Hill.

Romero-Medina, A. (2009). Evolución de la calidad y difusión de una revista científica española de Psicología: Anales de Psicología, 25 volúmenes anuales publicados (19842009). Anales de Psicología, 25(2), $181-198$. Recuperado de https://www.um.es/analesps/ v25/v25 2/01-25 2.pdf

Salgado, J. F. (2018). Transforming the Area under the Normal Curve (AUC) into Cohen's d, Pearson's rpb, Odds-Ratio, and Natural Log Odds-Ratio: Two conversion tables. European Journal of Psychology Applied to Legal Context, 10(1), 35-47. https://doi.org/10.5093/ ejpalc2018a5

Salvador-Oliván, J. A., y Agustín-Lacruz, M. D. C. (2015). Correlación entre indicadores bibliométricos en revistas de Web of Science y Scopus. Revista General de Información y Documentación, 25(2), 341 -359. http://dx.doi. org/10.5209/rev RGID.2015.v25.n2.51241

Vilariño, M., Amado, B. G., Vázquez, M. J., y Arce, R. (2018). Psychological harm in women victims of intimate partner violence: Epidemiology and quantification of injury in mental health markers. Psychosocial Intervention, 27(3), 145 152. https://doi.org/10.5093/pi2018a23 